\title{
The Lightwave programme and roadshow: an overview and update
}

Nicholas Wong, Matthew Posner, Pearl John

Nicholas H. L. Wong, Matthew T. Posner, Pearl V. John, "The Lightwave programme and roadshow: an overview and update," Proc. SPIE 9793, Education and Training in Optics and Photonics: ETOP 2015, 97932V (8 October 2015); doi: 10.1117/12.2223239

Event: Education and Training in Optics and Photonics: ETOP 2015, 2015, Bordeaux, France 


\title{
The Lightwave Programme and Roadshow: An Overview and Update
}

\author{
Nicholas H. L. Wong*a, Matthew T. Posnera, Pearl V. John ${ }^{\mathrm{b}}$ \\ aOptoelectronics Research Centre, University of Southampton, Southampton S017 1BJ, UK; \\ bPhysics and Astronomy, University of Southampton, Southampton SO17 1BJ, UK
}

\begin{abstract}
While optics and photonics are exciting disciplines with much research, industrial, and economic potential in the 21st century, this appreciation is only shared by a limited number of science, technology, engineering, and mathematics (STEM) experts, and there is a recognized STEM skills shortage. To widen the pool of talent, it is essential to expose students to optics and photonics throughout their education and particularly starting at a young age. The Lightwave programme, consisting of an interactive collection of photonics demonstrations and experiments targeted for primary school students, was thus created to facilitate this endeavor. The programme is run by doctoral students forming a team of "Lightwave ambassadors". All the demonstrations that comprise Lightwave can be easily integrated into a physics curriculum, enabling educators to generate more student interest and enhance the image of science through an interactive pedagogy. We provide a description of the programme at its initial inception, and report on the recent additions and updates that have brought about its success, moving from a purely outreach driven focus to engaging pupils with our own research. We also discuss our approach to ensuring that our team of ambassadors are from diverse backgrounds and use both male and female students as role models. Finally, we reflect on how evaluation methods to obtain feedback from our activities are key to Lightwave's sustainability and in improving the perception of optics and photonics.
\end{abstract}

Keywords: Optics education, Photonics outreach, Continuing education, Gender diversity in STEM.

\section{INTRODUCTION}

Since the publication of our Lightwave group's previous ETOP report in $2005^{1}$, a number of developments in the UK education sector have taken place. There have been changes in strategy and funding at the national level which have led to the development of local industrial partnerships and a more professional approach to delivering our Lightwave programme. This paper sets out to report on these changes.

We start by highlighting how the drive to tackle the photonics skills-shortage and gender imbalance in the UK education and industrial sectors has led to a culture change in the environment where our activity takes place. This change has caused Lightwave to move from a purely outreach driven focus to engaging pupils with our own research and incorporating methods of assessing our impact. A background of the Lightwave programme is given in $\S 2$. The Lightwave activities and evaluation methods are discussed in $\S 3$. In $\S 4$, we discuss the profile of the Lightwave ambassadors who act as role models to our audience. § 5 reports on a case study of our group's involvement at the International Year of Light 2015 (IYL 2015) opening ceremony to highlight our methods towards best practice. Finally, § 6 suggests some future work, and $\S 7$ concludes the report.

\subsection{The UK photonics skills shortage}

The UK government is enabling UK universities to respond to industrial skills shortages in areas which rely on photonics and optics by providing funding for universities to partner with industry ${ }^{2,3}$. There is a welldocumented skills shortage of physics and photonics students at the undergraduate and doctoral levels. "As photonics continues to expand, availability of people is increasingly an issue. However in parallel a more enlightened approach in vocational training is required to make sure those manufacturing lines can be expanded without delay" ${ }^{4}$ and effectively tackle the skills shortage caused by the growth of the photonics manufacturing industry. The USA has been tackling this shortage with vocational education for high school students since $1998{ }^{5}$, but we in the UK are only now starting to explore working with vocational institutions.

*nhlw1g12@orc.soton.ac.uk; phone +44 23 8059 5768; orc.soton.ac.uk

Education and Training in Optics and Photonics: ETOP 2015, edited by Eric Cormier, Laurent Sarger Proc. of SPIE Vol. 9793, 97932V · (c) 2015 SPIE, IEEE, OSA, ICO · doi: 10.1117/12.2223239 
The Institute of Physics outlines that in the UK, students least likely to study physics are girls, students from disadvantaged backgrounds, and students from certain ethnic minorities, e.g. Afro-Caribbean ${ }^{6}$. To address the corresponding skills shortage, universities have been working with a wider pool of potential students, expanding to these under-represented groups, using outreach activities to aid recruitment. In response to the 2012 increase in tuition fees to $£ 9,000$ per annum for UK students to attend UK universities ${ }^{7}$, the UK government's Office for Fair Access (OFFA) imposed on universities an Access Agreement: full fees could be charged if a percentage was spent on outreach with disadvantaged students ${ }^{8}$. Consequently, outreach with non-traditional groups is not just a strategy to widen the pool of students-and help to address skills shortages in industry - but is also a requirement for university educational funding. Subsequently, this has offered a new funding stream to develop photonics outreach programmes for universities to meet OFFA requirements and leverage professional standards of demonstrator training and remuneration.

\subsection{Women in optics and photonics}

A recent report ${ }^{9}$ highlights how all efforts over the last ten years to increase the number of female students studying physics have failed as a result of an unconscious bias; however, these are too numerous to explore in full in the scope of this article. One of the elements of the report argues that the language we use to describe what we do as scientists and engineers attracts men, not women, into the field. Generally, women and men self-identify with 'adjectives' and 'activity-oriented language', respectively, with the latter being much more commonplace in science education ${ }^{9}$. The report also observes that it is mothers who tend to discourage their daughters from working in physics and photonics because they believe that their daughters would not be happy working in male dominated fields. At the risk of over-generalizing, these observations about language use and behavior give us an opportunity to try something different to attract women into the field. The problem is complex, but we will show in $\S 4$ how a diverse team of Lightwave outreach ambassadors is trying to tackle this issue.

\subsection{Culture change: towards public engagement}

Universities in the UK generally rely on two different funding streams; teaching — via the intake of student feesand funding for research. The student recruitment agenda has always provided a motivation for outreach activities. However, UK researchers are now also expected to have "a strategic commitment to public engagement" and are commensurately "recognized and valued for their involvement with public engagement activities" 10 . As a result, they have a duty to demonstrate 'impact', i.e. to show that their research, which is enhanced by public engagement, makes a demonstrable contribution to society and the economy ${ }^{11}$. As a consequence, the purpose of photonics outreach is enriched as a vehicle to encourage a two-way dialogue between the public and academics about the university's actual photonics research, rather than the traditional practice of a one-way dissemination of information. The quality of the engagement is judging criteria for funding in UK HE through the Research Excellence Framework ${ }^{12}$. The activity must be designed accordingly to measure 'impact' through reach and delivery quality. We will describe how our programme has evolved to cater for this in $\S 3$. Before this, we offer a brief background to the Lightwave Programme and Roadshow.

\section{BACKGROUND TO THE LIGHTWAVE ROADSHOW}

\subsection{Synopsis}

The Lightwave Roadshow is an educational outreach programme run by doctoral (PhD) students of the University of Southampton, as part of the university's student-led Optics and Photonics Society (OPSoc) ${ }^{13}$. It is led by the society's Outreach Officer, a committee position that has come to be colloquially known as Lightwave Director, given its niche nature. Lightwave aims to teach children about light, and more importantly to get them engaged and interested in the broader fields of optics and science, from a young age. It consists of a wide variety of handson demonstrations to give children a basic understanding of optical phenomena encountered in everyday life, ranging from reflection and refraction to telecommunications. The demonstrations can be adapted to suit the various school year groups and also to develop specific aspects of the science curriculum. Lightwave runs workshops and demonstrations free of charge for schools at the University of Southampton and on request at schools in Southampton and the South East UK region, making around six visits a year; in addition, the programme travels to take part in scientific fairs and festivals. 


\section{$2.2 \quad$ Origins}

The roadshow traces its roots back to 1998, when the 1994-formed University of Southampton student chapter of The Optical Society (OSA), comprised of PhD students from the university's Optoelectronics Research Centre (ORC) and the School of Physics and Astronomy, was requested to develop simple optics experiments targeted at school children from 5 to 11 years of age. The experiments were constructed and demonstrated at a small number of local Southampton schools on an ad hoc basis. The encouraging reception received prompted the development of a permanent suite of optics experiments and kits featuring a spread of optical phenomena, and that could be reused as a standard inventory for multiple demonstrations and educational engagements ${ }^{14}$. These experiments would be demonstrated by OSA student chapter members and volunteer postgraduate students, either during oncampus workshops for visiting schools, or where we would visit a school and conduct the demonstrations there; the latter format alludes to the roadshow's namesake.

\subsection{Audience and delivery format}

Evolving from its original format of exclusively targeting primary school students-events for which we still run 2-3 times annually on average-the roadshow has expanded its audience and delivery to cater for older students between the ages of 11-18 in response to the development of the university's Widening Participation (WP) programme, "which has been designed to provide an education programme to engage and raise aspirations of gifted and talented students with the potential to enter higher education, but who may not have considered it" 15 . The workshops can be readily carried out in science classes, school halls, or gyms.

The mobile nature of the roadshow allows it to travel to festivals and engage with larger and more diverse demographics, extending to children beyond a school environment and adult members of the general public. The roadshow takes part annually in the Southampton Science and Engineering Festival ${ }^{16}$ held at the University of Southampton and during which the group engages with around 500 of the 3,000 people in attendance. The roadshow also has ad hoc presence at national festivals, such as the Big Bang Fair in March 2014, which saw over 1,000 people interact with Lightwave. In the past 18 months, our group has also conducted activities in partnership with the university's central Public Engagement for Research Roadshow ${ }^{17}$ to expand its teaching reach and raise awareness of the research in photonics and optoelectronics carried out by its ambassadors. A highlight of this partnership is the Bringing Science to Life school event where Lightwave travels to The Thomas Hardye School (THS), Dorchester, UK; over the course of one day, the group presents a 15-minute workshop on fiber optic communications and engages with over 300 students between the ages of 12-14.

\section{$2.4 \quad$ Funding}

Over the years, Lightwave has matured and grown in popularity and structure. In order to sustain its activities initially, including the development of new experiments, annual sponsorship amounting to US\$500 was obtained in the form of OSA outreach activity grants ${ }^{1}$. Sources of funding diversified with the evolution of the student chapter, such as the subsequent establishment of an SPIE student chapter and a European Physical Society (EPS) Young Minds section-the latter would last through to 2013. Combined with the existing OSA student chapter, these additional groups were consolidated into a student society called the University of Southampton Optical Society, and their respectively offered annual activity and outreach grants complemented the standing budget.

Following a rebranding of the society in 2015 into the present University of Southampton Optics and Photonics Society (OPSoc), which retains the original OSA and SPIE student chapters, Lightwave currently receives majority of its funding from OSA and SPIE student chapter activity and outreach grants. Examples of other sources of ad hoc funding include the Institute of Electrical and Electronics Engineers (IEEE) Photonics Society sponsored IYL2015 Chapter Challenge Grant ${ }^{18}$, and one-off sponsorships from the ORC ${ }^{19}$ and the University of Southampton.

A substantial amount of funding has been received as a result of the institutional strategy and culture change towards Outreach and Public Engagement alluded to earlier. More recent interaction with the university's WP programme has leveraged access to funding for the preparation and delivery of class-based activities on the subject of telecommunications for students between the ages of 14 and 18 years. The Engineering and Physical Sciences Research Council's Centre for Innovative Manufacturing in Photonics (EPSRC CIMP) has funded Lightwave's public engagement activities and we have correspondingly included coverage of manufacturing processes along with fiber optics and telecommunications applications in our activities to promote the research of the organization at the university. 


\subsection{The Light Express roadshow}

Lightwave has teamed up with a sister programme, the Light Express roadshow ${ }^{20}$, from Physics and Astronomy at the University of Southampton, as a result of obtaining joint EPSRC CIMP funding to reach large audiences. This roadshow visits schools and colleges in South England and has worked with over 60,000 pupils, teachers, and the public in the last 10 years. The Light Express features a dazzling laser light display created by powerful lasers not generally seen outside research laboratories. The show presents its audience with a series of fascinating visual demonstrations exploring the physics of light and the science behind the internet developed at the University of Southampton. The Light Express is aimed at large audiences-whole year groups-whereas Lightwave is aimed at smaller groups in both classes and fairs.

In response to its interaction with a wider audience and growing number of project partners, the activities proposed and requirements for ambassadors have evolved considerably. The next section describes updates on both these facets of the Lightwave Roadshow.

\section{ROADSHOW ACTIVITIES}

At its inception, the roadshow's activities were designed to teach children a little about light, and more importantly to get them engaged and interested in the pervasive world of optics and science with a variety of hands-on experiments. The experiments were supplemented by demonstrations, placing a strong emphasis on using practical and engaging tools to enthuse the audience. Through more recent exposure with other university bodies, we have observed an institutional level demand to demonstrate the impact of our programme. As a result, we have developed a set of evaluation tools in line with the programme's hands-on and engagement philosophies to measure and assess the impact of Lightwave. This section first reports on the coverage and state of the roadshow, with details on newer additions to our inventory. We then examine the elements of our activities that can be used to evaluate the success of the programme.

\subsection{Topics}

\subsubsection{A map of the Lightwave Roadshow}

The roadshow topics cover fundamental principles in optics, photonics, and optoelectronics. The activities can be broadly divided into the following sections: Mirrors \& Lenses, Sound \& Light, Optical Fibers \& Telecommunications, The Electromagnetic Spectrum, and Optical Illusions \& the Eye. The programme has a long heritage of purpose-made and 'off-the-shelf' equipment and the reader is referred to the Lightwave Roadshow's 2005 ETOP report ${ }^{1}$ for details on the equipment and the tailorability of activities to suit the various school year groups. The inventory is a combination of hand-built and commercial products; the latter items are listed in Table 1. An overview of the kits that would be used in an exhibition layout is presented in Figure 1.

Table 1. Commercially available Lightwave kits.

\begin{tabular}{|c|c|}
\hline Theme & Items \\
\hline \multirow{2}{*}{$\begin{array}{l}\text { Electromagnetic } \\
\text { Spectrum }\end{array}$} & Light Blox 21 \\
\hline & UV light 22 \\
\hline Sound \& Light & LED cube 23 \\
\hline \multirow{2}{*}{$\begin{array}{l}\text { Optical Illusions \& the } \\
\text { Human Eye }\end{array}$} & Floating illusions 24 \\
\hline & Praxinoscope 25 \\
\hline \multirow{4}{*}{$\begin{array}{c}\text { Optical Fibers \& } \\
\text { Telecommunications }\end{array}$} & Free-space video transmission ${ }^{26}$ \\
\hline & Fiber fountain 27 \\
\hline & Optical fibers 28 \\
\hline & Slinkies ${ }^{29}$ \\
\hline
\end{tabular}




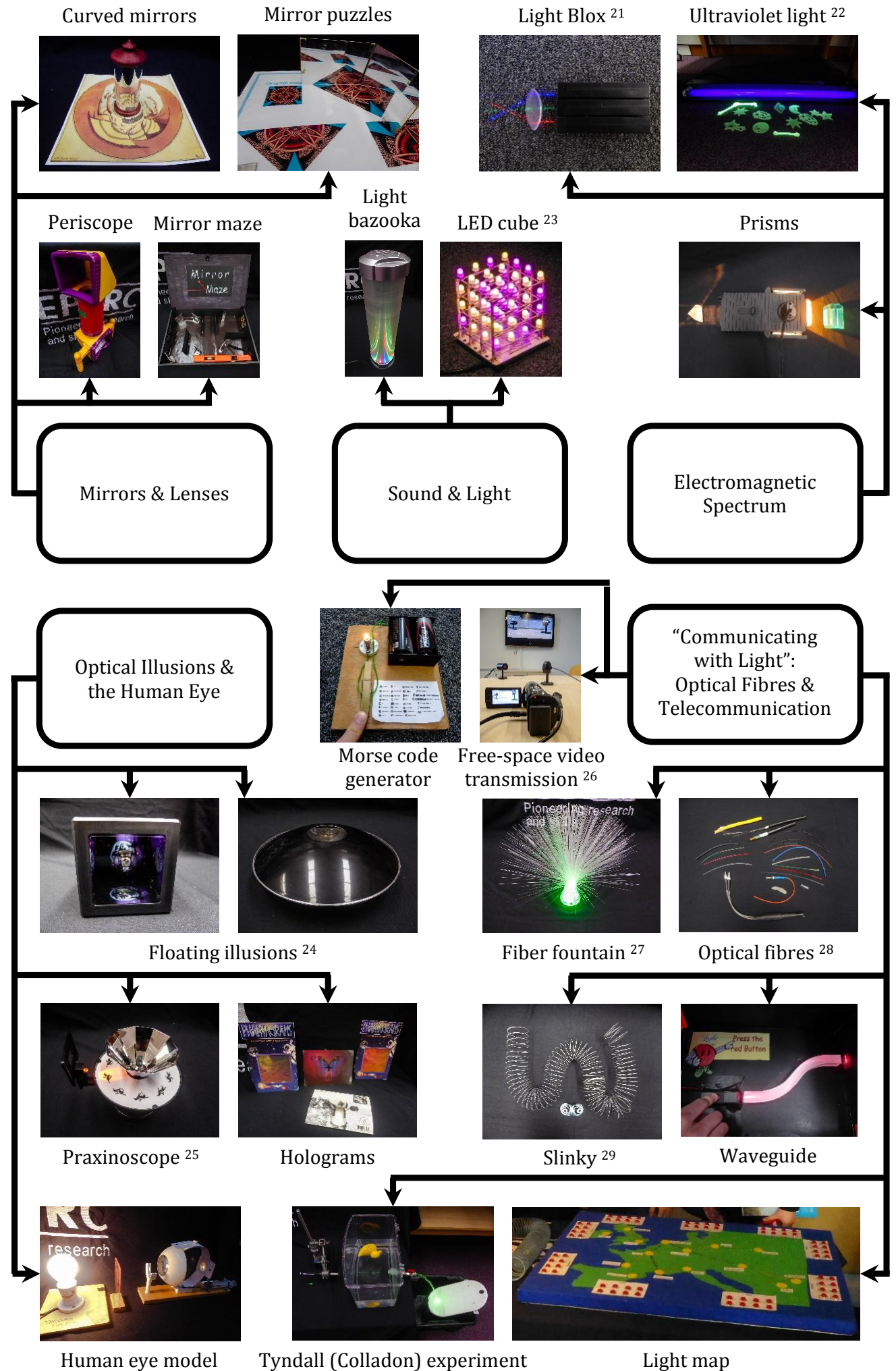

Figure 1. Lightwave experiments and kits, showing a sample exhibition layout. (In online version, pictures contain hyperlinks to commercially available equipment.) 


\subsubsection{Hands-on experiments}

Much of the Lightwave kit aims to encourage interaction and initiate experimental investigation on playing and working with light. For younger children in particular $(<11$ years old), the focus is given to exploration during the class-based activities. The hands-on experiments must therefore be robust. A number of professionally designed science kits are also available, such as the Optics Discovery kit ${ }^{30}$, the OSA suitcase ${ }^{31}$ and the Light Blox kit ${ }^{21}$, all of which have been incorporated into our core activities. They deliver a variety of optics experiments and provide comprehensive instructions to communicate scientific concepts to different age groups. Furthermore, introducing these kits to teachers allows educators to gain knowledge of readily accessible teaching equipment for further and more regular integration of optics and photonics into the science curriculum; the Lightwave ambassadors can then offer specialist advice on kit usage and methods to integrate practical classes to diversify children's optics education experience from a young age.

\subsubsection{Demonstrations}

Lightwave's experiments are complemented by accompanying demonstrations. All permit introductions to various photonics topics that can be tailored to suit different age groups. A specific example of how these demonstrations can be integrated into the school curriculum is the recent addition of the class-based session entitled "Communicating with Light" (developed independently from the highly acclaimed version for adults presented by Prof. P. Bayvel at the Royal Society in $2014{ }^{32}$ ). The class is an hour-long workshop suitable for age groups between 11-18 years to introduce students to photonics and demonstrate how light can be used as a communications method. The session aims to encourage discussion and prompt questions. A key challenge is to communicate a broad set of teaching objectives in an appropriate language to various age groups. The demonstrations can be customized for different age groups based on the expected knowledge acquired at different 'key stages' of the UK national curriculum ${ }^{33}$, or indeed as relevant to the country of origin of the reader.

The demonstrations must be selected to create rapport with the audience and contribute to the learning experience. The abstract and technical kit is not used for primary school students, where emphasis is laid on the more hands-on activities (e.g. Morse code machine) or the spectacular aspect of the show (e.g. laser bazooka, Tyndall experiment). Sessions for younger age groups (11-14) can complemented with a practical 'Morse code message' session, where participants are asked to send messages using Morse code and discuss advantages and impracticalities of the scheme. Older students (14+) will be presented more advanced kit where abstract concepts are required to appreciate their operation. For all age groups, it is essential to communicate the practical aspects of the underlying science and associated research as well as the qualities of the individuals presenting the topic. Demonstrating success of this is non-trivial and will be discussed more thoroughly in the following section.

\subsection{The evaluation activity}

With increasing demand to assess our impact, evaluation has become an essential part of the Lightwave activity. 'Impact' here refers to 'reach' and 'significance' of the outreach and public engagement activities. 'Reach' is defined as the number of people worked with, and 'significance' refers to the change in the knowledge, attitude, or behavior of the audience. We have integrated the collection of such data for evaluation purposes into aspects of our education programme. In the following section, we outline quantitative and qualitative evaluation tools and conclude with a discussion on ways in which we are working towards demonstrating evidence of impact.

\subsubsection{Quantitative data}

A typical metric for assessing impact 'reach' is the number of people with whom we interact. The simplest method of counting such interactions is by giving away branded items such as sweets, pens, bookmarks, or postcards, and tallying the number of items which have been distributed. This serves a dual function of quantifying impact as well as promoting the supporting organizations whose logos feature on the merchandise. It is possible to further distinguish between different audience demographics with targeted marketing or recruitment material, such as undergraduate study brochures for students or teacher information packs for educators. We caution however, that whereas this targeted merchandising provides conveniently segmented data pertaining to 'reach', it does not give any feedback on the education of the audience and whether or not the learning objectives have been achieved for the activity.

It is challenging to simultaneously educate, measure impact, and leave a positive impression of the interaction and activity. One particularly successful means employed is the use of handout diffraction gratings; these 'rainbow makers' are elegant items with which to engage children and teach them in a hands-on manner about the science of diffraction. Branded stickers can be added to these items to make them more special and raise awareness of supporting organizations (see Figure 2 for the IYL rainbow maker). We also provide activity 
information sheets for educators, with details about content and methods to integrate them into the curriculum. The exchange of business cards and contact details is another important metric to measure reach, provided that contacts are conscientiously followed up upon after the event.

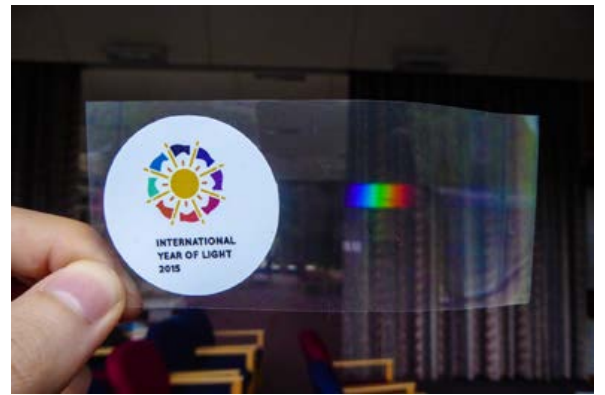

Figure 2. The IYL 2015 'rainbow-maker' to teach diffraction and measure the amount of engagement with the audience.

Finally, we measure interactions that truly demonstrate successful assimilation of photonics knowledge by using special rewards. The criteria are somewhat subjective, and we suggest, as a guideline, to have in place a documenting system (e.g. paper records, blogs, social media) for recording evidence of acquired knowledge. One particular example is a recent IYL poster competition initiated by the Lightwave Director in collaboration with a local secondary school. For this competition, 250 students between the ages of 14 and 15 were asked to produce posters on a technology of the IYL 2015; 20 students received IYL lapel pins for commended posters. A highly commended poster from an 'outstanding science communicator' is shown in Figure 3.



Figure 3. IYL special poster of the year, outstanding science communicator awarded to Ms. McIntyre, Year 9, The Thomas Hardye School, Dorchester, UK ${ }^{34}$.

By considering the total number of items handed out, one can work towards assessing the impact 'reach' of our programme in a purely quantitative manner. However, by further classifying these items into different categories, one can also evaluate the 'significance' of this interaction. We propose 4 levels of significance ratings:

1. General interaction with programme (pens, bookmarks, postcards)

2. Pitched interaction (teachers' packs, university brochures)

3. Scientific and educational interaction (diffraction gratings)

4. Demonstration of assimilation of knowledge or change of behavior (IYL 2015 lapel pins)

More concretely, at a science fair such as the Big Bang Fair (§ 2.3), a large number of branded merchandise (pens, postcards) will be handed out; however, they do not allow us to assess the change in the knowledge, attitude, or behavior of the audience, and they would have a low significance rating. At the other end of the spectrum, IYL 2015 lapel pins handed out to highly commended students taking part in the THS IYL 2015 poster competition mentioned above will have a high 'significance' score because there has been a clear demonstration of assimilation of knowledge. Figure 4 summarizes the reach and its significance of our Lightwave programme 
over the past 18 months according to these metrics. On top of this, we need to obtain feedback regarding the opinions of participants about their interactions with us, and as researchers, be willing to be influenced by our own public engagement experiences. We present methods of obtaining qualitative data in the next section.

\section{'Signifance' of the 'reach' of the Lightwave Roadshow}

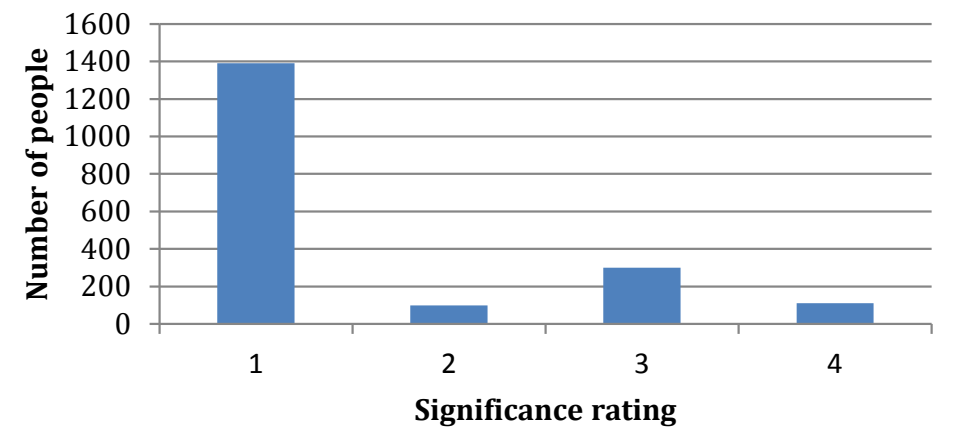

Figure 4. Towards quantitatively assessing the impact of the Lightwave Roadshow (October 2014 to June 2015).

\subsubsection{Qualitative feedback}

Qualitative data serves to measure the 'significance' of public engagement impact. Our methods of data collection necessarily differ between an event where Lightwave interacts with over 1,000 people (e.g. the Big Bang Festival at the National Exhibition Centre, Birmingham, UK, on March 16 2014), and a class-based activity with 20 students. At the root of either type of event however remains the need to give participants an enjoyable experience, impart scientific knowledge, and change beliefs and behavior where possible (i.e. to challenge negative stereotypes about who studies physics and photonics, and to inspire more students to consider studying these disciplines at university). To this end, we encourage written interaction concerning the activities, either through social media using tags and correct handles, or by using boards with post-it notes on what the audience has learnt. Figure 5 shows a response to one of the activities used at the Solent Skills Festival, Southampton, where students were asked to write on post-it notes about how they felt about the activity. The responses were later interpreted by a 15-year-old work experience student whom we believe was better equipped to translate the level of excitement of the audience. The results have helped assess the levels of enjoyment, though they fall short on assessing the assimilation of knowledge and do not offer feedback on the research.

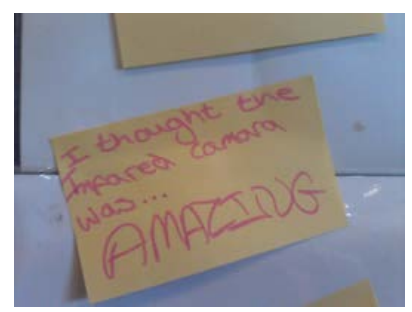

Figure 5. An example of qualitative feedback received using a post-it board to record audience interaction at the Solent Skills Festival, Southampton, UK, 22 October 2014.

The quality of the delivery must also be assessed by the success in communicating the scientific content of the roadshow; quizzes are a powerful tool to achieve this. In large fairs with a younger audience, a paper manuscript may be given to children to help them navigate around the experiments to find the answers themselves; this increases the interactive nature of the learning experience ${ }^{35}$, and a sample Lightwave quiz for 10 to 11 -year-olds can be found on our website ${ }^{36}$. A quiz may be graded and a student's level of understanding assessed; in addition, we can incorporate requests for feedback on our activity. For older children, it is possible to evolve towards online assessment platforms (such as Kahoot.it) that require access to mobile interfaces like smartphones and tablets. This increases the accessibility to quizzes beyond the classroom and provides tools for automating data collection and validation. Participation is further incentivized with quiz competitions and rewards (e.g. mugs, science kits, etc.), which has the knock-on effect of energizing the teaching experience. Online platforms offer 
direct feedback for students. The results also provide valuable feedback for us to improve and report on the activity.

\subsubsection{Pathways to impact}

To complete the evaluation, it is necessary for us to get our stories published in articles (in formats such as regional press, partner newsletters, webpages, and news sites) which increase the reach of our engagement activities - we note that a number of these articles are referenced herein. Close collaboration with public relations partners (e.g. in the university) is essential to assess the reach of the impact; for example, the ORC faculty newsletter Light Times reports on Lightwave's activities and is available both online and sent out in print to more than 1,300 people in 39 different countries. Social media offers a means to rapidly disseminate news of activities and measure the number of views and direct interactions. Interactions with Lightwave ambassadors, audiences, and project partners make it possible to expand the visibility of the activities, as do social media tags (e.g. geographic, events, hashtags).

\section{LIGHTWAVE AMBASSADORS}

The Lightwave Roadshow has traditionally been run by a team of volunteer PhD students ${ }^{1}$. Recently, new funding sources to develop, deliver, and measure impact of activities (see $\S 1$ ) have caused a shift away from this model. Today, Lightwave is organized around a team of 'ambassadors' and this section reports on the implications of this culture change for our programme.

\subsection{Ambassador profile}

A 'Lightwave ambassador' is, in broad terms, an individual who contributes towards the development, running, and recognition of the programme. The pool of candidates has been widened by our increased activity and interaction: members of staff from professional services now collaborate with PhD students to assist in the delivery of photonics education to young students and the general public. The larger and more diverse Lightwave group has expanded the reach, credibility, and visibility of our programme.

A key component of this group is the Lightwave Director. It is a strategic and managerial role that ensures the development and smooth running of the programme. The role oversees and feeds into 4 key segments, shown in Figure 6. Each segment requires a different set of skills, with potential personal and philanthropic benefits. The segments are non-exclusive and the group works at its best with efficient teamwork (with ambassadors contributing to each section); nevertheless, ambassadors can also be better suited to one section based on personal skills. The programme remains largely run by volunteers offering their time, though in response to the aforementioned culture change, financial remuneration for time is becoming more widely accepted as recognition of ambassador skills, in particular for the category of 'Science Communicators' that forms the largest population of the Lightwave programme.

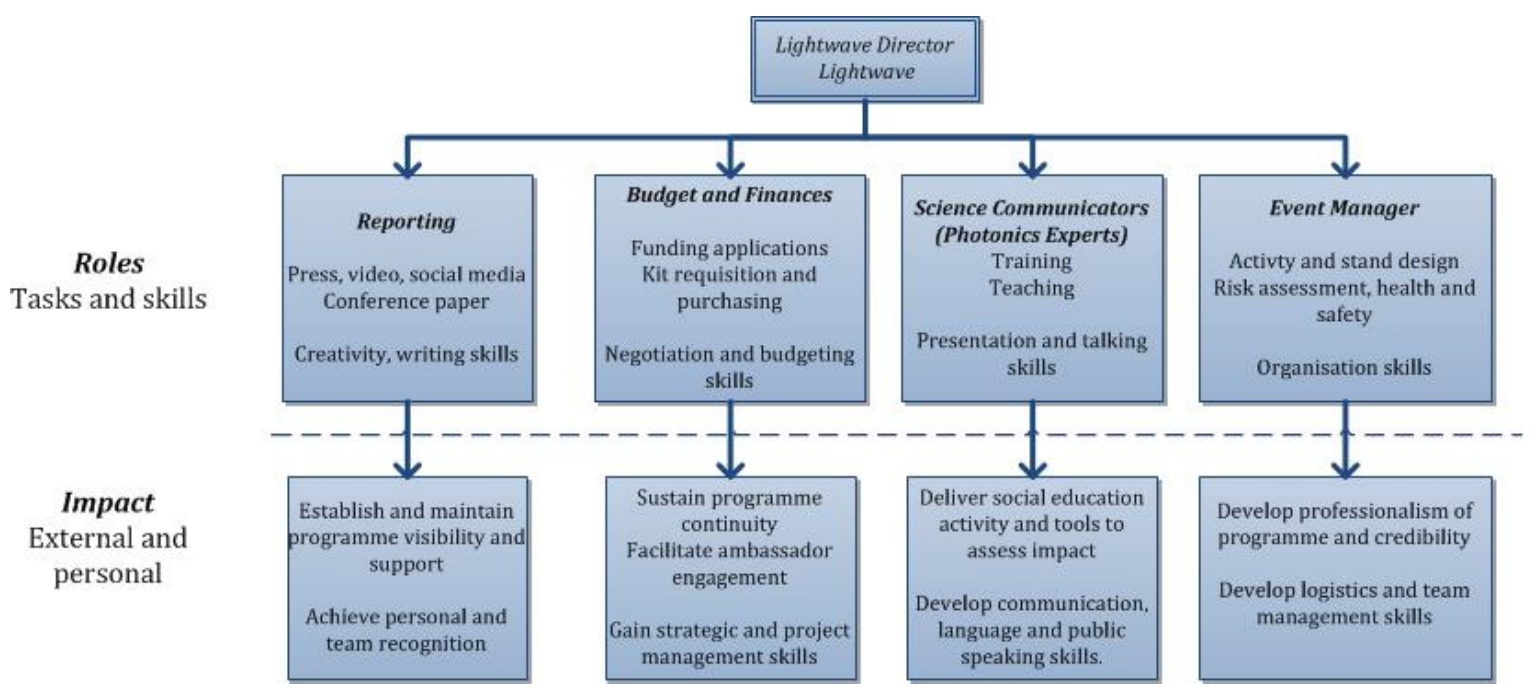

Figure 6. Organization of the Lightwave group, highlighting ambassador roles and impact on the programme. 


\subsection{Recruiting and training Science Communicators}

The traditional route to participate in Lightwave has been as a 'Science Communicator'. A typical profile would be a $\mathrm{PhD}$ student, with a desire to engage in presenting fundamental photonics to students and the general public; thus intrinsically, ambassadors are photonics experts. Since 2014, the programme, together with the greater OPSoc, has been introduced to new cohorts of ORC graduate students as part their induction programme and student-led workshops are run to induct ambassadors. The historical primary school activity does not necessitate further specific training; talking to young children is a daunting task, but with the emphasis being on facilitating exploration and playing with science, communication and language skills can be readily picked up on the job. However, with the evolution of our activity towards an older audience, further training is being sought after to improve our delivery and credibility for our audience; this is also partly a consequence of the added expectations of enhanced professionalism in representing project partners. There is a further drive to integrate the task of assessing engagement and impact into the programme, and ambassadors are now aware of the importance of two-way communication to facilitate this evaluation.

The ambassadors are also afforded the unique opportunity to speak about and advertise their own cutting edge research; a highly sought after skillset in their careers. The recognition of this skillset has further enabled remuneration of time used for demonstrating, which in turn has augmented the engagement of ambassadors and the activity of the programme. Finally, the ambassadors are role models for the students with whom they engage and the team must be diverse in nationality, ethnicity, age, and gender to better address the issues on the skills shortage and gender imbalance discussed in $\S 1$. A selection of recent photographs of ambassadors in action is shown in Figure 7.

(a)

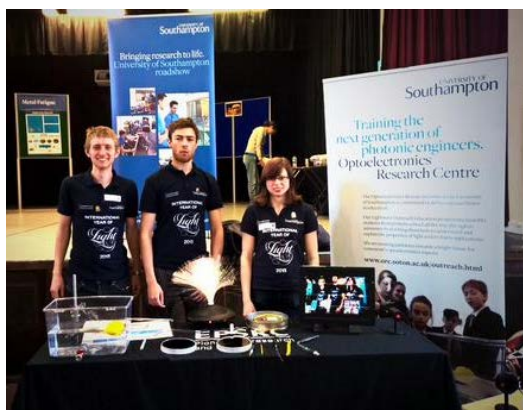

(c)

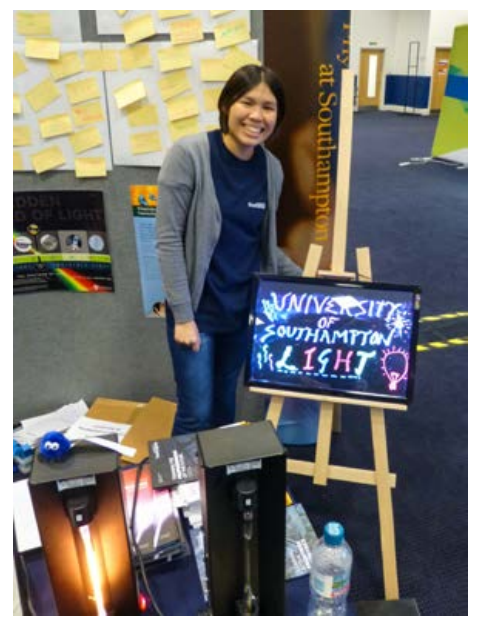

(b)

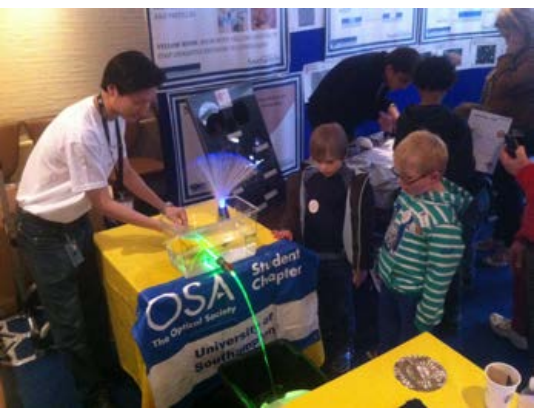

(d)

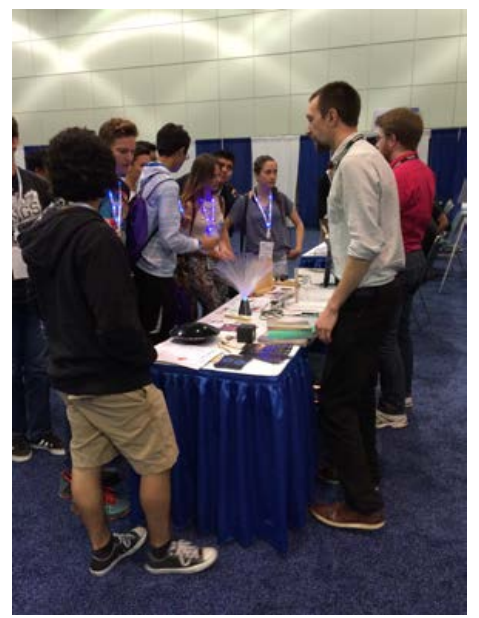

Figure 7. Lightwave ambassadors in action! (a) Matthew Posner, Nathan Soper, and Tina Parsonage 'Bringing Science to Life' at the THS Year 10 School Fair, April 2015. (b) Nicholas Wong demonstrating the Tyndall (Colladon) experiment at the University of Southampton Science and Engineering Day, March 2015. (c) Amy Tong 'lighting up' the Solent Skills Festival, October 2014. (d) Dr. Gregory Jasion and David Gray taking Lightwave abroad to the OFC conference, April 2014.

\subsection{Challenges}

The Lightwave programme has evolved considerably in size and activity, with our success leading to greater demand for shows. However, there remain a number of challenges that our group faces. An important one is the 
use of language. As referred to in $\S 1.2$, the use of 'male' language may have inhibited the gender diversification of the field of photonics. For example, co-author Matthew Posner would describe himself:

'Male' language

"As a scientist, I like problem-solving, organizing activities, and working with people."
'Female' language

"As a scientist, I am creative, organized, and a team player."

Using 'female' language is difficult, and both male and female scientists and engineers struggle to use it as it feels unnatural. The fact that it is uncomfortable underscores how alienating 'male' language must be for women to listen to and use when considering a career for themselves. We will continue to work both 'male' and 'female' approaches into our presentations and study if it makes a difference to the effect of our communication with our audience.

Another caveat of the programme is that it is mainly student led. Though the interaction with staff has increased our prominence and visibility, there is currently no permanent support staff or programme advisor to ensure continuity of the programme beyond the current Lightwave group. We have hitherto relied on the annual availability of a competent and committed (student) Lightwave Director, but that reliance is delicate due to the rapid turnover of $\mathrm{PhD}$ students. As a result, there is no long-term security, and longstanding goals and strategies are difficult to plan for and implement.

The group is also adapting in a period where the culture of public engagement of research and communicating science is evolving. There is a traditional expectation of giving 'free' time to public engagement and there are tensions as a result regarding the payment of Lightwave ambassadors. The drive towards 'free' public engagement threatens the prospect of remuneration for student development of communication skills through the 'Science Communicator' field of the programme; recognition of the development of photonics-expert science communicators will be a necessary step to achieve a sustainable solution.

\section{TOWARDS BEST PRACTICE: LIGHTWAVE AT THE OPENING CEREMONY OF THE INTERNATIONAL YEAR OF LIGHT AND LIGHT-BASED TECHNOLOGIES 2015}

In line with this year's IYL 2015 aim of raising global awareness of the ubiquity and utility of light in daily life 37 , and in seeking to mount a profile-raising initiative during this year of celebration, we carried out a project that ultimately sent a delegation of Lightwave ambassadors, public engagement staff, and staff and students from The Thomas Hardye School (THS) to attend the IYL 2015 opening ceremony in Paris, France, in January 2015.

The aim of the project was to internationally promote optics and photonics outreach and public engagement by Lightwave and the University of Southampton in general. The following sections outline the process, nature, and outcomes of the project.

\subsection{Sponsorship}

A total of $€ 5,000$ from the University of Southampton was secured for the project: $€ 2,500$ was first granted by the ORC to fund travel and logistic costs, and a further €2,500 was awarded by the Faculty of Physical Sciences and Engineering (FPSE) as a pledge to IYL 2015. The total amount established the university as a Gold Associate sponsor of IYL 2015. As a gold associate, the profile and recognition of the university, and the ORC in particular, is raised at the international level; an agreed-upon condition of the sponsorships.

\subsection{Delegation}

Comprising the team attending the opening ceremony were the following:

- 4 photonics PhD student Lightwave ambassadors from the ORC: Vinita Mittal, Martin Nunez Velazquez, Matthew Posner, and Nicholas Wong

- 1 Public Engagement leader from Physics and Astronomy, University of Southampton: Pearl John

- 1 physics teacher from THS: Geoffrey Reader

- $26^{\text {th }}$ Form students from THS: Katharine Bennett and Raphael Treccani-Chinelli 
The PhD students were selected on the basis of Lightwave ambassador experience and all hold positions in OPSoc's executive committee. The public engagement leader provided outreach advice and support, and complemented the team.

To include young students in this prestigious photonics event, 2 students at the $6^{\text {th }}$ Form level (17 years of age) from THS, a school with a well-established outreach relationship with Lightwave, were chosen to join the delegation. These students were identified as winners of a photonics-related essay competition adjudicated by our Lightwave Director. Joined by a senior physics teacher from the school, they would not only have the opportunity to gain exposure and become ambassadors at the global event, but more importantly, be able to share and propagate their experiences among their peers and spread the reach of inspiration.

\subsection{Presence}

The objective for attending the opening ceremony was not only to expose the delegation to an event attended by top minds in photonics and international diplomacy-and the valuable professional networking opportunities that would ensue-but also to gain international visibility for Lightwave, the ORC, and the university. This was achieved by running an exhibition booth showcasing experiments from our inventory, including the Tyndall (Colladon) experiment, the free-space video transmission kit, and the LED cube. Additional travel-easy new experiments were also demonstrated, such as a laser pointer and gelatin grid combination to show reflection and refraction. To address the issue of 'male' and 'female' language (see $\S 1.2$ and $\S 4.3$ ), we endeavored to consciously articulate both styles. Figure 8 shows various facets of the delegation and our interactions.

(a)

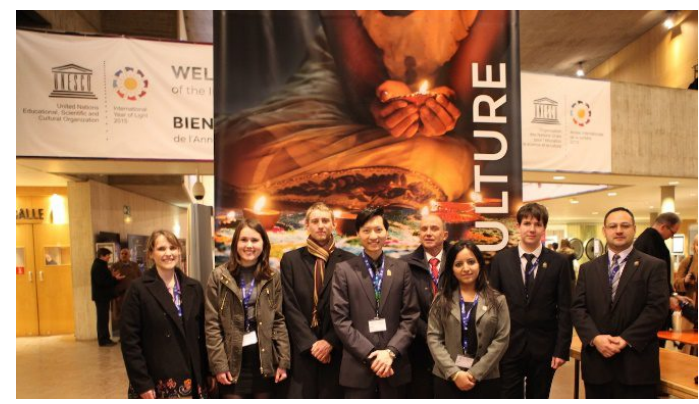

(c)

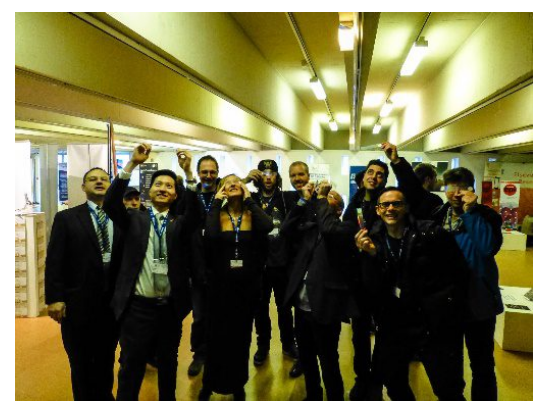

(b)

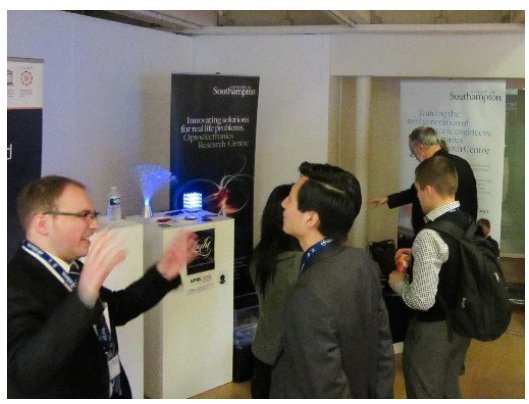

(d)

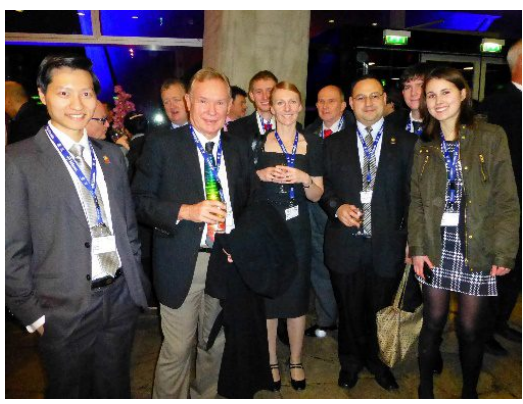

Figure 8. (a) Lightwave delegation at the IYL 2015 opening ceremony. (b) Ambassadors at our booth conversing with and demonstrating to event attendees. (c) Light artists examining diffraction gratings. (d) Delegation with ORC senior staff.

\subsection{Experience, outcomes, and publicity}

Our Lightwave exhibition booth was visited regularly by other event attendees throughout the 2-day ceremony. Visitors included academics and educators interested in our equipment and seeking either to complement their own local outreach programmes or start one following our example, photonics enterprise professionals curious about our outreach work, and light artists fascinated by the colorful illuminations of our LED cube, optical fiber fountain, and 'rainbow maker' gratings. These interactions produced valuable contacts, many of which generated follow-up requests for more information from us in the weeks after the event. One interaction even resulted in a subsequent addition to our OPSoc Seminar Series ${ }^{38}$.

As with any other Lightwave ambassador, the $6^{\text {th }}$ Form students were fully and actively engaged in demonstrating the experiments. Feedback obtained later from them was positive, with general sentiments about being inspired by the experience and an appreciation for the opportunity. 
To capitalize on publicity, social media, in the form of Facebook updates and Twitter tweets, were heavily utilized. An OSA Twitter contest 39 was won by the author for tweeting periodically about the event. Post-ceremony publicity was generated by personal blog articles ${ }^{40,41}$ as well as the press ${ }^{42}$.

\section{FUTURE WORK}

The EPSRC CIMP ceases to exist from the end of June 2015 when Public Engagement and Outreach funding also ends. The close collaboration with the Light Express roadshow will hopefully continue with further funding from researchers working with manufacturing applications of photonics and optics. If that is indeed the case, the content of our public engagement activities needs to continue to move towards emphasizing the importance of photonics and optics in manufacturing, rather than just in telecommunications.

In July 2015, Lightwave will perform at the Royal Horticultural Show (RHS) at Tatton Park 43 to a non-scientific audience. Lightwave has partnered with Garden Designer Helen Elks to consult on the design of a show garden influenced by fiber optics research. Visitors, press, and members of the RHS will visit the garden and meet Lightwave ambassadors to discuss the technology of holey fibers. This is considered an excellent opportunity to reach new audiences who are unfamiliar with science outreach activities. Women with children-and grandchildren-are an important demographic to target to challenge the stereotype that a career in physics (and by extension, photonics and optics) is only for men.

The Lightwave group is seeking to establish a regular calendar of university-based and regional events to maintain the running of its programme in the long term. The teaching programme is constantly evolving, notably in response to the changing research foci of the ambassadors. For instance, we are working to expand the photonics teaching coverage to include specific areas in optoelectronics; an IEEE Photonics Society grant was awarded in April 2015 to develop this aspect. To overcome the problem of fast turnover of ambassadors intrinsic of a student organization, we are currently in discussion with academic staff at the university to establish a staff Lightwave advisor for continuity, and to develop further training to raise awareness of language profiles, continuing to research and test methods to best evaluate our programme.

\section{CONCLUSIONS}

Over the last 10 years, the Lightwave Roadshow has matured into a successful and dynamic outreach and public engagement programme, bringing optics and photonics to young students and the general public. Its initial goals, which involved promoting these fields among primary school students to attempt to tackle the industry skills shortage, as well as training ambassadors in communication skills, have expanded to include helping to widen participation at the university and encourage more women to take up photonics. There has also been an academic culture change that places increased emphasis on public engagement, rather than general outreach events, with a need to evidence impact from these activities. These changes have collectively directed the evolution of the roadshow, and today, it is carried out in a varied number of formats for different purposes, each showcasing a comprehensive assortment of equipment and experiments that are complemented by a diverse and effective team of ambassadors. We have increased our reach and visibility by taking Lightwave abroad, and will continue to explore more ways to develop and improve this vibrant programme.

\section{ACKNOWLEDGEMENTS}

The authors wish to thank the many University of Southampton ambassadors who have supported the Lightwave Roadshow throughout the years, and extend our gratitude to all our partners, collaborators, and sponsors, notably: ORC, FPSE, and the University of Southampton; EPSRC CIMP; OSA; SPIE; EPS; the IEEE Photonics Society; and the IYL 2015 steering committee. 


\section{REFERENCES}

[1] Foreman, H. D., Parmigiani, F. R., Roelens, M. A., and Simpson, R. E., "The Lightwave Roadshow," Proc. SPIE, Educ. Train. Opt. Photonics Conf., 140-148 (2005).

[2] Standen, D., "Training the photonics pioneers of the future," EPSRC CIMP, 2010, <http://www.cimp.soton.ac.uk/about.html> (5 May 2015).

[3] "Photonic Integration and Advanced Data Storage," University of Glasgow, <http://www.gla.ac.uk/schools/engineering/research/centrefordoctoraltraining/> (5 May 2015).

[4] Silvera, I., "UK Universities Siphon £8.1m Into Laser and Cloud Computing Training to Tackle Science Skills Shortage," International Business Times, 29 May 2014, <http://www.ibtimes.co.uk/uk-universitiessiphon-8-1m-into-laser-cloud-computing-training-tackle-science-skills-shortage-1450395> (6 May 2015).

[5] John, P. V., Miller, N., and Shanks, R., "Public education and the electro-optical industry: a strategic partnership in creating photonics classes for high school students," Proc. SPIE 3831, Sixth Int. Conf. Educ. Train. Opt. Photonics (2000).

[6] "Raising Aspirations in Physics: Recommendations from a review of research into barriers to STEM participation for students from disadvantaged backgrounds,"Institute of Physics, (2014).

[7] Weale, S., "Payback looms for first $£ 9,000$ tuition fee students," the guardian, 29 December 2014, $<$ http://www.theguardian.com/education/2014/dec/29/tuition-fees-tertiary-students-ifs-struggle-

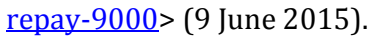

[8] "About the Office for Fair Access (OFFA)," OFFA, <http://www.offa.org.uk/about/> (5 May 2015).

[9] MacDonald, A., “'Not for people like me?' Under-represented groups in science, technology and engineering," WISE, 1-32 (2014).

[10] "Concordat for Engaging the Public with Research,", RCUK, <http://www.rcuk.ac.uk/RCUKprod/assets/documents/scisoc/ConcordatforEngagingthePublicwithResearch.pdf> (5 May 2015).

[11] "RCUK Impact Requirements: Frequently Asked Questions," RCUK, March 2011, <http://www.rcuk.ac.uk/RCUK-prod/assets/documents/impacts/RCUKImpactFAQ.pdf> (6 May 2015).

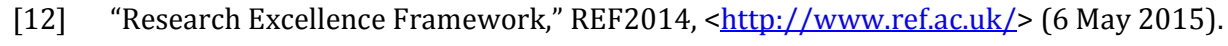

[13] "University of Southampton Optics and Photonics Society," < http://osa.orc.soton.ac.uk/> (5 May 2015).

[14] Bricchi, E., Baggett, J. C., Guilhot, D. A., and Musgrave, I. O., “The lightwave road show," Proc. Educ. Train. Opt. Photonics, OSA Tech. Dig. Ser. (2003).

[15] "Learn with US: The University of Southampton's Widening Participation programme,"

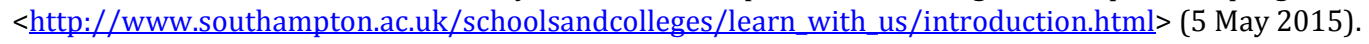

[16] "Southampton Science and Engineering Festival," University of Southampton, <http://www.southampton.ac.uk/per/university/festival/index.page> (5 May 2015).

[17] "Bringing research to life. University of Southampton Roadshow," University of Southampton, <http://www.southampton.ac.uk/per/university/roadshow.page> (5 May 2015).

[18] "IEEE Photonics Society Volunteer Opportunities," IEEE Photonics Society, $<$ http://photonicssociety.org/sites/default/files/IEEE Photonics Society Volunteer Opportunities 1-615.pdf> (7 June 2015). 
[19] "Outreach: International Year of Light 2015," Optoelectronics Research Centre, 2015, <http://www.orc.soton.ac.uk/outreach.html > (5 May 2015).

[20] "The Light Express," Physics and Astronomy, University of Southampton, <http://www.lightexpress.soton.ac.uk/> (6 May 2015).

[21] "Light Blox Kit: International Year of Light Edition," Laser Classroom, <http://store.laserclassroom.com/tech-light-lab-light-science-kit> (5 May 2015).

[22] "Maplin 20W Fluorescent UV Blacklight," Maplin, <http://www.maplin.co.uk/p/maplin-20w-fluorescentuv-blacklight-l28bb?gclid=CjwKEAiA0uGmBRDwj7mE1vLICYSIADxH160GJSUt7425tvhhKxCg0mJuFP7SIXopsr8M8qDwvECMRoCCXLw wcB> (7 June 2015).

[23] "Rainbow Cube Kit (Assembled)," Seeed Studio, <http://www.seeedstudio.com/depot/Rainbow-Cube-KitAssembled-p-998.html> (7 June 2015).

[24] “Magic Money Bank," Hawkin's Bazaar, <http://www.hawkin.com/magic-money-bank> (7 June 2015).

[25] “Animation Praxinoscope," 4M Kidz Labs, <http://www.amazon.co.uk/Great-Gizmos-4142-KidzLabs/dp/B000H5V07U> (7 June 2015).

[26] "Laser Communication Kit," Pasco, <http://www.pasco.com/prodCatalog/SE/SE-8793 lasercommunication-kit/> (7 June 2015).

[27] "Fibre Optic Ice Crystal Lamp," The Glowhouse Ltd, <http://www.glow-sticks.org/Fibre-Optic-Ice-CrystalLamp.html> (7 June 2015).

[28] “Optical Sample Kit," Industrial Fiber Optics, <http://i-fiberoptics.com/educationaldetail.php?id=15700\&cat=kits-projects\&sum=> (7 June 2015).

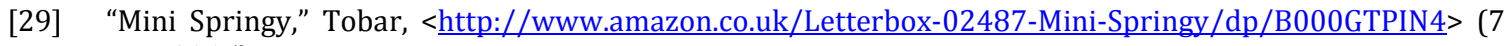
June 2015).

[30] “Optics Discovery Kit,” Edmund Optics Inc., <http://www.edmundoptics.com/optics/optical-lenses/lenskits/optics-discovery-kit/1865/> (5 May 2015).

[31] "Optics Suitcase," The Optical $\quad$ Society,

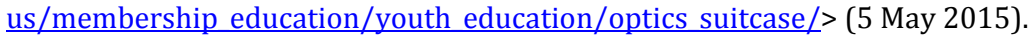

[32] Bayvel, P., "Communicating with light," The Royal Society, 27 October 2014, <https://royalsociety.org/events/2014/10/clifford-paterson/> (5 May 2015).

[33] "National curriculum in England: science programmes of study," GOV.UK, $<$ https://www.gov.uk/government/publications/national-curriculum-in-england-science-programmesof-study> (5 May 2015).

[34] "International Year of Light and Light-Based Technologies Poster Competition," The Thomas Hardye School, April 2015, <http://www.thomas-hardye.net/pages/news/2015/0415/IYLPoster/IYLPoster.php> (5 May 2015).

[35] McLoughlin, C. and Reid, N., "Seachange: Design of online quiz questions to foster deep learning," Proc. ASCILITE, 843-846 (2002).

[36] Posner, M. T., "Lightwave Quiz," <http://lighwave-dev.orc.soton.ac.uk/sites/lighwavedev.orc.soton.ac.uk/files/Year 6 Lightwave Quiz.pdf> (9 June 2015). 
[37] "Resolution adopted by the General Assembly on 20 December 2013. 68/221. International Year of Light and Light-based Technologies 2015," United Nations General Assembly, UN Doc A/RES/68/221 (2014).

[38] Barrera, E., "PhotonTransfer.com and the XPhoton Open Innovation Challenge for the IYL2015," University of Southampton Optics and Photonics Society (2015).

[39] "Want to win some International Year of Light (IYL) swag?" The Optical Society, 2015, <http://www.osa.org/osaorg/media/osa.media/IYL/IYL_Tweets_-_Opening_Ceremony_4.pdf> (7 June 2015).

[40] Bennett, K., “IYL 2015 Opening Ceremony: Highlights and Reflections," 22 January 2015, $<$ https://fightthepatriarchy.wordpress.com/2015/01/22/iyl-2015-opening-ceremony-highlights-andreflections/> (5 May 2015).

[41] Wong, N. H. L., "Light is Now: the International Year of Light Opening Ceremony," 31 January 2015, <https://spectroverse.wordpress.com/2015/01/31/light-is-now-the-international-year-of-light-openingceremony/> (9 June 2015).

[42] “International Year of Light Delegation," Southern Daily Echo, 19 January 2015, < http://www.dailyecho.co.uk/news/11733717.International Year of Light delegation/> (7 June 2015).

[43] "RHS Flower Show Tatton Park," Royal Horticultural Society, <https://www.rhs.org.uk/showsevents/rhs-flower-show-tatton-park> (5 May 2015). 